\title{
El rol del docente en la singularidad educativa
}

\section{The Role of the Teacher in the Educational Singularity}

\author{
Eduardo Caballero Ardila \\ ecaballero@media-x.tv n https://orcid.org/0000-0003-3034-3357 \\ Universidad Monteávila, Venezuela
}

Fecha de recepción: 6 de abril de 2020

Fecha de aceptación: 27 de abril de 2020

Fecha de publicación: 30 de junio de 2020

Favor de citar este artículo de la siguiente forma:

Caballero Ardila, E. (2020). El rol del docente en la singularidad educativa.

AULA, Revista de Humanidades y Ciencias Sociales, 66 (2), 27-34.

DOI:https: //doi.org/10.33413/aulahcs.2020.66i2.134

\section{RESUMEN}

La profesión docente se encuentra ante el reto de formar individuos que están permanentemente impactados por los contenidos audiovisuales a través de la red de internet y por avances tecnológicos permanentes; todo en un entorno donde el cambio es una constante. La comprensión de las características conductuales de esta población es fundamental para el éxito del proceso enseñanza-aprendizaje.

La singularidad es reconocida como un proceso que apunta al desarrollo exponencial de la tecnología, e impulsa nuevos modelos sociales y culturales. En consecuencia, el salto del quehacer educativo desde la tiza y el pizarrón hasta el mismo oficio en la robótica, rebasa la capacidad de adaptación de los docentes para acometer con éxito la transmisión de competencias y conocimientos a comunidades que, culturalmente, piensan y se comportan de manera distinta. Este trabajo propone abordar la concepción de los estudiantes con visión de audiencias educativas, con características distintas en cuanto a su poder atencional, consumo de medios de formación y plataformas que le son propias. Igualmente analiza el rol del docente para la formación de nuevos individuos en la singularidad desde el punto de vista tecnológico y desde su práctica pedagógica. Concluye con el análisis del reto para lograr la transformación de los objetivos pedagógicos en las naciones con economías menos sustentables.

\section{Palabras clave: Singularidad, audiencias educativas, educación virtual,} formación docente, plataformas educativas.

\footnotetext{
ABSTRACT

teaching profession is faced with the challenge of training individuals who are permanently impacted through the Internet network by audiovisual content and by permanent technological advances, all in an environment where change is constant. Understanding the behavioral characteristics of this population is essential for the success of the teaching-learning process.
} 
Singularity is recognized as a process that points to the exponential development of technology, and drives new social and cultural models. Consequently, the leap from the educational task from the chalk and the blackboard to the profession itself in robotics, goes beyond the ability of teachers to adapt to successfully transmit the skills and knowledge to communities that culturally think and behave different. The work proposes to approach the conception of the students with vision as educational audiences, who have different characteristics regarding their attention power, consumption of training means and platforms that are their own. It also analyzes the role of the teacher for the formation of new individuals in the singularity from the technological point of view, as well as from their pedagogical practice, and concludes with the analysis of the challenge to achieve the transformation of pedagogical objectives in nations with less sustainable economies.

Keywords: Singularity, educational audiences, virtual education, teacher training, educational platforms.

\section{La singularidad}

El abordaje de las audiencias educativas en la modernidad, es un reto académico que trasciende la apropiación de las herramientas tecnológicas, y requiere la comprensión de grupos etarios, que a través del impacto de contenidos globalizados disponibles en la red adquieren conductas académicas mediatizadas por el cortoplacismo audiovisual, por la sobreabundancia de información y por las nuevas necesidades de una sociedad. En palabras de Bauman (2004), «el ingrediente crucial de este cambio multifacético es la nueva mentalidad a corto plazo, que vino a reemplazar a la mentalidad a largo plazo. Adicionalmente, los métodos tradicionales de apropiación del conocimiento, ceden paso frente a la necesidad de formación para una sociedad que incentiva, pero por otro lado está obligada, a vivir en el entorno de un desarrollo exponencial del conocimiento mediatizado por el avance de la tecnología y de la ciencia». Cabero (2001), indica que las relaciones entre la sociedad y las tecnologías son bidireccionales, de forma que la sociedad influye para la creación y potenciación de determinadas tecnologías y al mismo tiempo, las tecnologías impulsan determinados modelos sociales y culturales.

Raymond Kurzweil (2012), en su obra La Singularidad está cerca. Cuando los humanos transcendamos la biología, desarrolla el concepto de «la singularidad», que es el momento en el que en opinión del autor desaparecen las fronteras entre la inteligencia biológica y la inteligencia artificial. Lo muestra como el momento cuando el desarrollo de tres tecnologías: genética, nanotecnología y robótica, conllevará a la liberación del ser humano de sus cadenas biológicas y a la consagración de la inteligencia como el fenómeno más importante de nuestro universo. El autor explica que el crecimiento exponencial de la tecnología se expresa por la ley de los rendimientos acelerados (Kurzweil, 2012). Se piensa que el primero que mencionó el término «singularidad» fue John von Neumann, americano de origen austrohúngaro conocido sobre todo como padre de la Teoría de Juegos y autor de la Arquitectura de von Neumann para ordenadores (Witt 2008).

Caravaca (2016), indica que la singularidad, coincide con un nuevo concepto más allá de la humanidad que denomina «transhumanidad», y que consiste en el empleo de las nuevas ciencias y tecnologías para mejorar las capacidades físicas y mentales con el fin de corregir aspectos de la condición humana como las enfermedades o incluso la propia mortalidad.

La singularidad entiende al humano como un software (inteligencia, emociones, consciencia y 
procesos biológicos) y un hardware (capacidad y resistencia) que puede perfeccionarse (Serra 2016). Mediante la genética y la medicina regenerativa, el ser humano va a ser capaz de superar condicionamientos biológicos como el envejecimiento, así como la limitación de sus capacidades intelectuales. Mediante la nanotecnología el ser humano manipulará cada vez más la materia átomo a átomo, creando así un horizonte de abundancia material capaz de cubrir todas las necesidades de la humanidad, y por último, el desarrollo de la robótica desembocará en la creación de inteligencia artificial fuerte, es decir, inteligencia artificial de nivel humano. Esta inteligencia permitirá continuar con la aceleración del desarrollo tecnológico y la fusión entre ser humano y máquina, lo cual permitirá que el sustrato humano deje de ser biológico y pase a ser tecnológico (Kurzweil 2012).

Sea que se cumpla cabalmente, o no, la predicción de Kurzweil, lo cierto es que los métodos para la apropiación del conocimiento en una sociedad distinta deben ser distintos, en consecuencia el ejercicio docente también debe cambiar. En palabras de Viñals y Cuenca (2016), «una sociedad en la que el aprendizaje ya no es una actividad individual, sino un continuo proceso de construcción de redes. Aprender es el equivalente a abrir una puerta a una nueva forma de percibir y conocer, donde nuestra mente debe adaptarse al entorno», de manera que una nueva sociedad requiere de un nuevo tipo de docente. Fernández y Fernández (2016) desarrollan el concepto de la formación de alumnos denominados como alumnos de la «Generación Tecnológica»o «Generación Z».

\section{Características}

\section{de las audiencias educativas}

Este trabajo plantea la sustitución del término «alumno», en correlación al sinónimo de discípulo, aludiendo a las personas que reciben enseñanza mediante la única guía de un profesor, en la esfera de una escuela, colegio o universidad, y propone su sustitución por «audiencia educativa», un término que ofrece una visión más amplia y que reconoce la interacción, así como las características de una comunidad que se interrelaciona, comunica, y aprende, apoyada o complementada por medios audiovisuales y a través de la red. Una modificación semántica que permite entender y abordar a los educandos desde una perspectiva más amplia en relación a sus costumbres y características.

$\mathrm{Y}$ es en relación a las características de estas audiencias donde Núria Miró, citada por Moreno (2019), indica que el perfil del estudiante cambiará en los próximos tres lustros. Indica Miró que será un alumno con muchas más posibilidades de acceso a fuentes de conocimiento, con una mentalidad más universal y menos localista, protagonista indiscutible de su aprendizaje, un ciudadano global que busca a través del aprendizaje un modo de responder a alguna necesidad del entorno.

Tres características conductuales deben ser resaltadas de las nuevas audiencias educativas.

La primera característica de este tipo de audiencias, es que son «no lineales» y privilegian los modelos asincrónicos, que no requieren de un horario específico para consumir contenidos como los audiovisuales o los videojuegos, al igual que los contenidos educativos. En la industria de la televisión esto ha supuesto lo que se denomina «la muerte del programador»,es decir, el cese de esa figura que en una mezcla de arte y ciencia indicaba a las audiencias audiovisuales el día y la hora de visualización de los diferentes contenidos audiovisuales. En el sector educativo esto no es distinto, la apropiación del conocimiento en el salón de clase como punto de encuentro entre el docente y sus alumnos, para el desarrollo de las estrategias de enseñanza-aprendizaje, considerado como una formalidad necesaria para la obtención de un título educativo, es complementada, y en ocasiones suplementada por 
contenidos educativos que se consumen de manera no lineal por las comunidades educativas. En otras palabras hablamos del proceso de conversión de las actuales escuelas en «escuelas-red», para adaptarse a la estructura de la sociedad-red, con contenidos diseñados para una red global y diseñados para un mundo globalizado (Jaurata, Imbernon et.al 2012).

La segunda característica es el tiempo atencional de las audiencias educativas que adquieren comportamientos similares al de las audiencias en los medios radioeléctricos y en las redes sociales, con un poder atencional mucho más corto al de las clases magistrales divididas en horas académicas, sin interrupciones, iniciando y finalizando a la hora establecida. El modelo de orientación de la atención de Posner (1980) ${ }^{1}$, establece que la atención se puede dividir en tres redes definidas anatómica y funcionalmente, las cuales interactúan entre sí. La primera es la red de alerta, que es la encargada de ayudarnos a mantener el estado de vigilancia y activación durante el día; posteriormente la red de orientación espacial, que se activa cuando orientamos nuestra mente a algún evento, y finalmente la red de atención ejecutiva, que está relacionada con todos los procesos que nos ayudan a regular nuestra conducta y cognición, y se encarga de inhibir información distractora o conductas habituales cuando no son necesarias; también de monitorear nuestra conducta de manera que podamos adaptarnos a las circunstancias que nos presente el entorno. Investigadores como Stuart y Rutherford (1978), Bunce (2010), o Francisco Mora

${ }^{1}$ Para los interesados en el modelo de Posner, sugerimos la lectura de Funes, M.J. \& Lupíañes, (2003). J. La teoría atencional de Posner: una tarea para medir las funciones atencionales de Orientación, Alerta y Control Cognitivoy la interacción entre ellas.

${ }^{2}$ Las teorías centrales de la economía de la información, relacionan los sistemas de información y la economía. Este fenómeno ha sido desarrollado por autores como George Akerlof, Michael Spence y Joseph Stiglitz.
(2013), han validado la tesis del corto espacio de la red de atención ejecutiva en contraposición con el esquema tradicional fundamentado en la clase magistral. En el nuevo mundo de la economía de la información ${ }^{2}$, las principales plataformas tecnológicas de comunicación lo han entendido y capitalizado. Twitter, en ciento cuarenta caracteres; Instagram en videos que no superan un minuto de duración, o el desarrollo de las historias de corta duración en plataformas como Facebook.

La tercera característica de las audiencias educativas, es su capacidad multiplataforma. De nuevo, el esquema docente que busca capitalizar la totalidad del poder atencional en una sesión académica, compite contra medios audiovisuales como la televisión abierta, la televisión por suscripción, la televisión digital terrestre, -en los países que pasaron el apagón analógico-, los video juegos, los medios OTT -como Netflix, Amazon prime o HBO-, y muy especialmente contra las redes sociales. Todo lo anterior ha modificado la concepción del pensamiento social, permitiendo que este se encuentre en un proceso de inestabilidad, que hace un llamado obligatorio a la flexibilidad de los procesos, o de lo contrario, se incurre en el error de construir pensamiento muy efímero, con muy pocas posibilidades de renovarse y ajustarse a las nuevas tendencias (Mejía García \& Acosta Pérez, 2019).

De manera que mientras el docente despliega estrategias docentes para abordar los contenidos educativos, existe la posibilidad, o la casi certeza, de que sus alumnos estén consumiendo algún otro tipo de contenido en dispositivos como los teléfonos o las tablets, razón por la cual, el esfuerzo de mantener su atención es doblemente exigente.

\section{Un nuevo docente para la singularidad} «El tiempo adquiere historia cuando la velocidad de movimiento a través del espacio se 
convierte en una cuestión de ingenio, imaginación y recursos humanos» (Bauman 2004). La única constante en el desarrollo de la humanidad es el cambio, de manera que la figura del docente y su formación estuvo, está, y estará mediatizada por los cambios del entorno.

Planteada la figura del maestro como un líder, que inspira pero que igualmente se recicla frente a los nuevos tiempos, surge la inquietud sobre la orientación, los enfoques del rol y en consecuencia la formación del docente en el marco del desarrollo de las tecnologías de información y comunicación. ¿Cuál es el rol del docente en la singularidad? ¿Cómo adaptar la formación de los docentes a esta nueva realidad? ¿Dónde queda la necesidad de crear un individuo con capacidad de creación y de motivación? ¿Hasta dónde llegarán estos cambios?, ¿Cómo el avance tecnológico afectará estos planteamientos? Fugett (2005), se cuestiona ¿Cómo podremos integrar equipos humanos con talento y pericia (pool de talento, tanques de pensadores) que generen soluciones creativas y propongan innovaciones y las ejecuten en relación con la educación de las sociedades futuras?

Abordando la formación del docente, Fuguett (2005), analiza cómo hasta los 70 la formación era conductista, asimilándolo a un técnico especialista en aspectos moleculares del proceso con concepción tecnocrática del curriculum basado en la disciplin. La renovación desde los años setenta, con una visión humanista, un docente dotado de motivación y recursos internos para promover y mejorar el ambiente educacional. Y luego, el desarrollista, que ve la formación del docente como un líder, un solucionador de problemas, donde su negocio son las transacciones.

El docente preparado para formar a nuevas comunidades, experimenta cambios en sus estrategias metodológicas tal y como indica Cooperberg (2002), en «cómo se va a ir construyendo en procesos desconstructivos y reconstructivos, pero de la mano de procesos interactivos que realice el sujeto, tanto en el conocimiento mismo, como con los demás participantes».

En palabras de Tedesco (2012), esto se logrará desarrollando en el docente un discurso educativo con significado y sustentación. Una educación que estará en el centro de las estrategias de construcción de sociedades justas a través de dos grandes pilares: en términos sociales y políticos «aprender a vivir juntos», y en términos cognitivos «aprender a aprender.»

De manera que el reto docente aborda dos instancias, una tecnológica y otra de visión pedagógica. Desde la acera de la tecnología, el docente debe estar en capacidad de planificar por competencias en plataformas distintas a las del aula de clase, como lo es el caso de la educación virtual. En ella se ve obligado a producir su propio contenido educativo, grabando, iluminando correctamente, recogiendo de manera adecuada el audio y post produciendo hasta su inserción en la red, todo eso en función a las competencias requeridas por sus audiencias educativas. Estos docentes además, son capaces de interactuar a través de las distintas plataformas de comunicación -como Zoom, Skipe, Zello-, de aulas virtuales -como Classroom, Office o Moodle-, y de los MOOC -como Udemy, Tutelluz o Coursera-, o incluso los propios que toda institución educativa debe tener. Todo lo anterior con un currículo personalizado a la medida de las necesidades de cada estudiante y donde destacan habilidades personales y competencias.

Este nuevo abordaje requiere de las instituciones educativas un proceso de adaptación al mundo virtual, que pasa por la selección e incorporación de la tecnología adecuada, del entendimiento de las características y comportamiento de las audiencias educativas, y por la formación de los colaboradores docentes en aspectos como el conocimiento técnico de la educación virtual, el uso de las distintas plataformas, técnicas de oratoria en video conferencias, planificación para el ambiente virtual, así como técnicas de producción audiovisual. 
Desde la acera de la visión pedagógica, la respuesta del docente en la singularidad parece estar en la cita de Schubert (2000), que combina tres descriptores: educador, filósofo y líder, abordando elementos como la metafísica que permite al docente asumir realidades múltiples (políticas, económicas); la epistemología (acercamiento al conocimiento; la axiología (formularse interrogantes sobre lo deseable); la ética, que basa a la educación para lograr un desarrollo intelectual y moral; la estética, construyendo y recreando espacios para el aprendizaje; la lógica, fortaleciendo espíritu crítico y constructivo; la política, honrando la diversidad; la democracia, y la teodicea.

Fuguett (2000), plantea en su obra El perfil del docente. La vuelta a la inspiración-como una de las ideas centrales-, la figura del docente como líder, definiendo como tal un educador que es capaz de pensar, crear, diseñar, resolver, interactuar, usar, manejar, producir y comunicar información, trabajar, estudiar, construir visiones en equipo, autoevaluarse, compartir y comprometerse, amar, querer y sentir.

\section{¿Tecnología para quién?}

Conquistar el éxito en la formación de individuos para un mundo que no existe es una combinación de visión, esfuerzos; pero también de recursos. Es allí donde se abren brechas entre países desarrollados, con economías sólidas, y el resto de las naciones sin economías sustentables. El avance tecnológico imparable, así como su aplicación beneficiosa en el sector educativo, no es una propuesta con resultados lineales. El éxito está atado a la disponibilidad de recursos para acceder a ese desarrollo tecnológico, lo que supone una desventaja para el desarrollo educativo en las naciones más desfavorecidas.. Las universidades y los centros de divulgación científica son igualmente punto estratégico de desarrollo. La U.S. National Science Foundation (NFS) destaca en su informe del año 2020, cómo las 15 economías de mayor desarrollo económico a nivel mundial concentran y monopolizan los artículos científicos en las áreas del saber humano científico, tecnológico y humanístico. Un reto para quienes no acumulan ni producen riqueza a la par de estas naciones.

\begin{tabular}{|c|c|c|c|c|c|c|}
\hline RANK & $\begin{array}{l}\text { REGION, COUNTRY } \\
\text { OR ECONOMY }\end{array}$ & 2008 & $\begin{array}{l}\text { RAGE ANN } \\
2018\end{array}$ & $\begin{array}{c}\text { GROWTH RATE } \\
\text { 2008-18 (\%) }\end{array}$ & $\begin{array}{c}2018 \text { WORLD } \\
\text { TOTAL (\%) }\end{array}$ & $\begin{array}{l}2018 \text { CUMULATIVE } \\
\text { TOTAL (\%) }\end{array}$ \\
\hline- & World & $1,755,850$ & $2,555,959$ & 3.83 & - & - \\
\hline 1 & China & 249,049 & 528,263 & 7.81 & 20.67 & 20.67 \\
\hline 2 & United States & 393,979 & 422,808 & 0.71 & 16.54 & 37.21 \\
\hline 3 & India & 48,998 & 135,788 & 10.73 & 5.31 & 42.52 \\
\hline 4 & Germany & 91,904 & 104,496 & 1.28 & 4.08 & 46.61 \\
\hline 5 & Japán & 108,241 & 98,793 & -0.91 & 3.87 & 50.47 \\
\hline 6 & United Kingdom & 91,358 & 97,681 & 0.67 & 3.82 & 54.29 \\
\hline 7 & Russia & 31,798 & 81,579 & 9.88 & 3.19 & 57.49 \\
\hline 8 & Italy & 56,157 & 71,240 & 2.41 & 2.79 & 60.17 \\
\hline 9 & South Korea & 44,094 & 66,376 & 4.17 & 2.60 & 62.87 \\
\hline 10 & France & 66,460 & 66,352 & -0.02 & 2.60 & 65.47 \\
\hline 11 & Brazil & 35,490 & 60,148 & 5.42 & 2.35 & 67.82 \\
\hline 12 & Canada & 53,296 & 59,968 & 1.19 & 2.35 & 70.17 \\
\hline 13 & Spain & 44,191 & 54,537 & 2.13 & 2.13 & 72.30 \\
\hline 14 & Australia & 37,174 & 53,610 & 3.73 & 2.10 & 74.40 \\
\hline 15 & Iran & 17,034 & 48,306 & 10.99 & 1.89 & 76.29 \\
\hline - & EU & 528,938 & 622,125 & 1.64 & 24.34 & - \\
\hline
\end{tabular}

$\mathrm{Eu}=$ European Union

Fuente: U.S. National Science Foundation (2020). 
Una brecha que no para de crecer y que debe ser detenida con el apoyo de organismos multilaterales de asistencia educativa y tecnológica, y adicionalmente haciendo que estos países vuelquen su mirada hacia la importancia del desarrollo educativo como la mejor estrategia de largo plazo para construir prosperidad ciudadana.

\section{Conclusiones}

El ejercicio docente tiene como reto formar a ciudadanos para desempeñarse en una sociedad globalizada, interconectada y transversalmente mediatizada por tecnologías que no le son propias a una generación que ha abordado ese desarrollo como migrantes digitales. Adicionalmente despliega sus esfuerzos para conducir el proceso enseñanza aprendizaje con audiencias educativas que se conducen de una manera que no le es propia a los docentes en la actualidad.

\section{Referencias}

Bauman, Z. (2004). Modernidad Líquida. Fondo de Cultura Económica de Argentina S.A. Buenos Aires. Argentina.

Bunce DM, Flens EA, Neiles KY. (2010). How long can students pay attention in class? A study of student attention decline using clickers. $J$ Chem Educ 87: 1438-1443.

Cabero Almenara, J. (2001). La sociedad de la información y el conocimiento, transformaciones tecnológicas y sus repercusiones en la educación. En Sociedad de la información y educación. Florentino Blázquez Entonado (Coordinador). Conserjería de educación, ciencia y tecnología. Junta de Extremadura. España.

Caravaca, L. (2016). La Singularidad: hacia el futuro de la humanidad. Eduskopia. Recuperado de https://eduskopia.com/la-singularidad-hacia-el-futuro-de-la-humanidad/

Cooperberg, A. (2002). Las herramientas que facilitan la comunicación y el proceso de enseñanza-aprendizaje en los entornos de educación a distancia. Revista de Educación a Distancia, ISSN 1578-7680, No. 3

Fernández Cruz, F. y Fernández Díaz, M.J. (2016). Los docentes de la Generación $\mathrm{Z}$ y sus competencias digitales. Revista Comunicar. No. 46. v. XXIV. Recuperado de www.revistacomunicar.com
La respuesta pasa por un proceso de formación docente, pero muy especialmente por redescubrir la visión educativa en un mundo que es y será distinto. Los Estados tienen como desafío interpretar los tiempos y planificar un proceso desde una óptica distinta que no se soluciona con cambios curriculares, sino con un proceso de planificación estratégica en cada país, en el cual se aprovechen las fortalezas y se potencien las oportunidades, mientras se atacan las amenazas y se corrigen las debilidades del sector educativo.

La singularidad es un proceso indetenible, entendido como un conjunto de transformaciones profundas en los sectores educativos, culturales, económicos y sociales.

La incógnita es el tiempo y la manera de abordarlos como sociedad, y el sector educativo está en la obligación junto a las familias, de formarse para potenciar a los adultos del futuro
Fuguett, A. (1999-2000). El perfil del docente. La vuela a la inspiración. Revista Educación. Revista para el Magisterio. Nro. 184.

Fuguett Smith, A., Vivas, D. y Sosa, P. (2005). La visión de la universidad en tiempos de cambios. Discusión pedagógica. SAPIENS vol. 6 no. 2 Caracas dic. 2005. Recuperado de FUGUET/LECTURAS/3La\%20visión\%20 de $\% 201 \mathrm{a} \% 20$ universidad $\% 20$ en $\% 20$ tiempos $\% 20$ de $\% 20$ cambios.webarchive

Jaurata, B., Imbernón, F., Álvarez, J.M et al. (2012). Pensando en el futuro de la educación. Colección críticas y fundamentos. Editorial Grao. España.

Kurzweil, R. (2012). La singularidad está cerca. Cuando los humanos transcendamos la biología. Lola Book. Berlin.

Mejía García, D. y Acosta Pérez, B. (2019). Avances tecnológicos modernos y sus implicaciones en el pensamiento social. AULA Revista de Humanidades y Ciencias Sociales. 65 (5), 29-37. http://dx.doi.org/2636.2236/ AULA.2019.012

Mora, F. (2013). Neuroeducación. Lo que nos enseña en cerebro. Alianza Editorial. España.

Moreno, A. (2020). Así será la escuela en 2030. Edición digital del periódico El Mundo. España. Recuperado de https://www.elmundo.es/espana/2014/10/21/54455b9f22601d22738b458e.html 
National Science Foundation (2020). Publications Output: U.S. Trends and International Comparisons https:// ncses.nsf.gov/pubs/nsb20206/publication-output-by-region-country-or-economy

Posner, M.I. (1980). Quarterly Orienting of Attention Journal of Experimental Psychology. Experimental Psychology Society. Recuperado d https://journals.sagepub.com/doi/ $10.1080 / 00335558008248231$ ? cookieSet $=1$

Serra, M.A. (2016). Singularidad tecnológica. Recuperado de http://www3.uah.es/proyectogse/glosario/frontend/ web/index.php?r=termino\%2Fview\&id=121

Schubert, W. (2000). Educator-philosopher-leader: conceptual framework. University of Illinois at Chicago \& Council of Teachers Education. Chicago Ill. afs/2002.

Stuart, J. \& Rutherford, R.J. (1978). Medical student concentration during lectures. Lancet
Tedesco, J.C. (2012). Escuela y sociedad en el siglo XXII. Pensando en el futuro de la educación. Colección críticas y fundamentos. Editorial Grao. España.

Viñals, A. y Cuenca, J. (2016). El rol del docente en la era digital. Revista Interuniversitaria de Formación del Profesorado, vol. 30, núm. 2, agosto, 2016. Asociación Universitaria de Formación del Profesorado. Zaragoza, España. Recuperado de https://www.redalyc.org/ pdf/274/27447325008.pdf

Witt, F. (2008). Singularidad tecnológica y transhumanismo: cuando la ciencia se encuentre con la ficción. Cordoba, España. Recuperado de https://www. academia.edu/5264382/SINGULARIDAD_TECNOLÓGICA_Y_TRANSHUMANISMO_CUANDO_LA_CIENCIA_SE_ENCUENTRE_CON_LA FICCIÓN

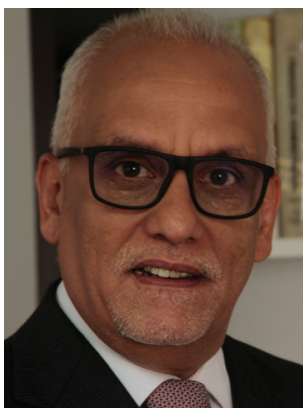

Eduardo Caballero Ardila

Doctorado en Licenciado en Contaduría Pública de la Universidad Católica Andrés Bello, Programa Avanzado de Gerencia del Instituto de Estudios Superiores en Administración «IESA», Magister en Comunicación y Educación de la Universidad Autónoma de Barcelona y Doctorante en Educación, Políticas Públicas y Profesión Docente (Universidad Pedagógica Experimental Libertador y UNESCO). Presidente de la Escuela Audiovisual Mediax, y Profesor en la Universidad Monteávila de Caracas. Autor del libro Dirección y Gestión de Empresas Audiovisuales (Editorial Universo de letras) e investigador en el ámbito de la educomunicación. Blog: https:// eduardocaballeroardila.wordpress.com 